Military Technical College Kobry Elkobbah, Cairo, Egypt May $16-18,2006$

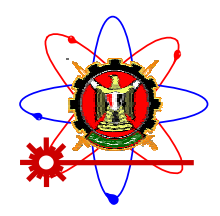

$3^{\text {nd }}$ International Conference on Engineering Mathematics and Physics (ICMEP-3)

\title{
Experimental investigation of vircator by DC discharge
}

\author{
TAHA H.F., Msc ${ }^{*}$, ALLAM.M.A.A., Prof ${ }^{*}$, EL-SHAER M.A., Prof ${ }^{* *}$, \\ and MASOUD M. M., Prof.
}

\begin{abstract}
A vircator powered by DC power supply has been designed, operated and tested. The discharge is between mesh anode and disc cathode. It has been found that the discharge is in the up-normal glow discharge and also follows Child law. Experimental studies showed that the optimum spacing between the mesh anode and disc cathode is $3.5 \mathrm{~mm}$ at the adopted spectrum. The discharge current variation with the spacing between electrodes has a peak value of $20 \mathrm{~mA}$ at $3.5 \mathrm{~mm}$ for the same applied voltage and gas pressure. Several microwave frequencies radiated from the system have been observed. The radiated power for the different frequencies has a peak value at nitrogen gas pressure of 0.7 Torr and $3.5 \mathrm{~mm}$ electrodes spacing.
\end{abstract}

\section{KEY WORDS: Vircator, DC-Discharge, Microwave Frequency}

\section{I-INTRODUCTION}

One of the classic applications of electron beams is the generation of coherent electromagnetic radiation. The development of sources of radiation in the high power microwave portion is within electromagnetic spectrum but it is classified as plasma devices. However, an impressive increase in the power and operating frequencies of these sources has taken place in the last few decades [1]; due to progress in a new branch of physics that can be called relativistic high frequency electronics which is very tighten to plasma microwave generation physics [2]. The progress results from three main developments. Firstly the use of higher voltages and currents in conjunction with conventional microwave device concepts, such as klystrons and magnetrons. Secondly the development of new device concepts that rely specifically on very high currents, such as vircators. And thirdly is the research into fast wave devices, such as gyratrons and free electron lasers, that make direct use of relativistic effects, and extrapolate to higher powers at shorter wavelengths than conventional devices [3]. The main mechanism of vircator is the formation of virtual cathode and electron beam for the cathode, which penetrate the formed plasma to generate microwave. Several authors [4-7] have presented theoretical studies of vircator. The vircator consists of mesh anode and disc cathode in plane geometry each, and some other coaxial geometry are used. Virtual cathode is unstable region of negative potential which reflects electrons back into the source between the real and virtual cathodes [8].

\footnotetext{
${ }^{*}$ E.A.F. (M.T.C.) Cairo

${ }^{* *}$ Head of Physics and Mathematics Department, Faculty of Engineering Zagazig University ***Professor, Plasma Physics Department, Atomic Energy Authority, Cairo.
} 
Oscillations in the virtual cathode and harmonic generation associated with that region and reflection of coherent electrons cause the higher frequency components. Oscillations of electrons between the virtual cathode and mesh anode generate microwaves with frequency lower than those of the electron beam interaction with the plasma [9]. Possible mechanisms for excitation of electromagnetic waves in virtual cathode systems were Studied by Kitsanov et.al [10]. They found that the microwave efficiency of doublegap vircator can be higher than that of single gap. Simulation of the vircator behaviors and energy exchange between the electron beam and the RF field, for single and double-gap in 1-D and 3-D, were presented using the KARAT fully electromagnetic PIC code. Simulation of dual-coaxial vircator, without external magnetic field had been carried out by Yang and Ding [11]. They found that by applying a voltage pulse of $500 \mathrm{kV}$, the emitted microwave power will reach a peak value of $2.5 \mathrm{GW}$, with $11 \%$ efficiency. In this study 0-50 kV DC power supply is connected to a vircator which consists of a stainless-steel mesh anode and disc cathode. Experimentally studies are presented for the generation of microwave from DC discharge under different conditions, and also to measure the output microwave power and frequency. Electrons emitted from the cathode will be accelerated towards the anode mainly without collisions. Hence it will cross the anode and undergo collisions outside the mesh anode. Such collisions will produce plasma sheath acts as a virtual cathode.

\section{II-EXPERIMENTAL SETUP}

The virtual cathode oscillator (vircator) is developed and designed. The schematic diagram of its electrical circuit arrangement is shown in figure (1). The output of high voltage DC power supply is connected to the two electrodes where the mesh anode was at positive potential with respect to the disc. The disc cathode is 4-cm-diameter cathode made of copper connected to screw bolt long head that screws into a nut used to fasten the cathode with $1 \mathrm{~mm}$ pitch for one complete turn of the screw bolt. This system is used to adjust the distance of the cathode inside the housing between $0.5 \mathrm{~mm}$ to $6.5 \mathrm{~mm}$. This controls and adjust the anode- cathode gap distance( A-K ), since the mesh anode is at fixed position in the housing of the vircator. The mesh is surrounded by two flanges centered inside a 12-cm inner diameter housing $17-\mathrm{cm}$ longitudinal distance made from stainless steel. The metallic steel mesh anode is made from wire with thickness $1 \mathrm{~mm}$ and with 60 holes $/ \mathrm{m}^{2}$ with overall diameter $12 \mathrm{~cm}$. Two ports are made at the mid axial sides of the vircator with $5 \mathrm{~mm}$ diameter and $2 \mathrm{~mm}$ thickness. The ports are connected to the vacuum pump system and monitoring thermocouple vacuum gauge. To adjust the pressure inside the cylindrical housing needle valve is used to calibrate continues flow of working gas.

Calibrated voltage and current meters have been used for the diagnostics of the vircator discharge characteristics. The detection of the generated microwave frequencies are carried out by AGILANT frequency analyzer. The frequency range of this study is $200 \mathrm{MHz}-2 \mathrm{GHz}$.A linearly polarized wide band log-periodic antenna is designed and constructed from lightweight corrosion- resistant aluminum to afford this frequency band, another type of antenna is used up to $13 \mathrm{GHz}$.

\section{III-EXPERIMENTAL RESULTS}

The variation of the discharge current with the voltage between the mesh anode and disc cathode at pressure $5 \times 10^{-1}$ Torr is shown in figure (2). The I-V curve showed that the discharge is similar to that of the abnormal glow discharge between two solid electrodes. The difference between this discharge and the abnormal glow DC discharge is the neutral atoms which are ionized outside the two electrodes. Since the electrons are accelerated by space charge, it has been found that the I-V curve followed Child law [12], Ia $\mathrm{V} 3 / 2$

where: $V$ is the accelerating voltage and $I$ is discharge current.

The dependence of the breakdown voltage on the gas pressure $(p)$ multiplied by the electrodes spacing (d) is shown in figure (3). The curve is similar to Paschen curve where at low pd higher voltage is required for 
breakdown. At left hand side of Paschen curve when pd increased the breakdown voltage decreased, till reach a minimum value. The minimum breakdown voltage about $450 \mathrm{~V}$ at $\mathrm{Pd} 1.75 \mathrm{Torr}$. $\mathrm{mm}$. For the right hand branch the breakdown voltage increases slowly with the increase of pd i.e. the ionization cross section increases, therefore electrons need more energy to ionize the neutral atoms. The operation condition has to be in the left hand side of Paschen curve [13]. The maximum electron beam current depends on the mesh anode and disc cathode (A-K) gap distance. The A-K gap spacing has been changed from $0.5 \mathrm{~mm}$ up to $6.5 \mathrm{~mm}$, and measure the discharge current, keeping the gas pressure at $5 \times 10^{-1}$ Torr, and constant applied potential at $2.5 \mathrm{Kv}$ DC voltage, Figure (4). It has been found that the discharge current increases with increase of gap distance until it reach its maximum, $20 \mathrm{~mA}$, at spacing of $3.5 \mathrm{~mm}$. The increase of the discharge current from $1 \mathrm{~mm}$ up to $3.5 \mathrm{~mm}$ may be due to the increase of the electrons velocity while the decrease of the current after $3.5 \mathrm{~mm}$ can be due to the increase of electrons collision probability with neutrons, which reduce the electrons velocity.

Figures $(5,6,7)$ are samples of the measured variation of the emitted frequencies at different conditions which is in agreement with the electrical parameters of the discharge. Radiated microwave amplitude versus $\mathrm{A}-\mathrm{K}$ gap of the vircator has been investigated at different frequencies from $200 \mathrm{MHz}$ up to $500 \mathrm{MHz}$ for working gas pressure $5 \times 10^{-1}$ Torr figure (8). It has been found that the maximum amplitude at $d=3.5$ $\mathrm{mm}$ for all observed frequencies. Figure (9) shows the radiated microwave amplitude variation with working gas pressure for A-K spacing of $3.5 \mathrm{~mm}$. It has been found that radiated microwave amplitude voltage had a peak value at $7 \times 10^{-1}$ Torr, for all the investigated frequencies.

\section{IV-Conclusion}

Vircator with stainless- steel mesh anode and cupper disk cathode is presented. The vircator is powered by DC power supply $50 \mathrm{Kv}$. The I-V characteristics shows that the discharge is within the up normal glow discharge and follows Child law. Pd relation with discharge voltage is obtained which is typically Paschen curve. It is found that electron beam discharge current varies with the anode cathode $(A-K)$ spacing, where it reached its maximum value at $3.5 \mathrm{~mm}$. The microwave output is dependent on the anode cathode (A-K) gap distance, where the optimum spacing which gives the maximum output microwave power is of the order $3.5 \mathrm{~mm}$ over frequency range $0.2-0.5 \mathrm{GHz}$. The variation of working gas pressure shows a peak radiated wave amplitude potential at 0.7 Torr.

\section{ACKNOWLEDGEMENTS}

The authors like to thank Dr .EL MENHAWY A.A. Dr. HAMAD .G .M and Dr. SHANAN .S.S for guidance and valuable scientific help.

Also, they would like to thank AZOZ A., MAHMOUD B., ELWEY E. and EL-BASHARI A., for the help that have given especially with the Vircator testing and technical support .

\section{References}

[1] Taylor. C.D. and giri .D.V., High- Power Microwave Systems and Effects Taylor\&Francis (1994)

[2] Benford I, Swegle.J . High - Power Microwaves. Artech House, Chapter 9. *2(1992)

[3] Granatstein VI, Alexeff I. High - Power Microwave Sources. Artech House ; Chapter 13,14 (1987)

[4] Jiang W,, Wollverton K., Dickons J., and Kristiansen M. IEEE Trans. Plasma Sci., vol. 27, $1538(1999\}$ 
[5] Alyokhin B.V., Dubinov A.E., Selemir V.D., Shamro O.A., ShibalkoK.V.,Stepanov N.V., and Vatrunin V.E. IEEE Trans. Plasma Sci., vol. 22, 945 (1994).

[6] Chen X., Dickens J., Mankowski J., Hatfield L.I., Choi E.H., and Kristiansen M. IEEE Transactions on Plasma Science vol. 32, 1799 (2004).

[7] Jiang W., and Kristiansen M., Phys. of Plasmas, vol.8,1187(2001)

[8] Choi.E.H, Choi.M.C, Choi.S.H, Song.K.B, Jung.Y, Seo.Y.H, Shin.H.M, Uhm.H.S, Lim.D.W, Kim.C.H, Lee.J.M and Ahn.J.W. IEEE Trans. Plasma Sci., vol. 30,1728 (2002).

[9] Benford J., Price D., Sze H., and Bromley D., J. Appl. Phys., vol. 61, 2098(1987).

[10] Kitsanov S.A., Klimov A.I., Korovin S.D., Kurkan I.K., Pegel I.V., and Polevin S.A. IEEE Transactions on Plasma Science vol. 30, 274 (2002).

[11] Jiang W., and Ding Wu. . IEEE Trans. Plasma Sci., vol. 32, 1187 (2004).

[12] Roth J.R. "Industrial Plasma Engineering" Institute of Physics Publishing, vol. 2 page 417 (2001)

[13] Saudy M.A. , Eatah A.I., and Masoud M.M. Fizika A 14, 3,225(2006) 


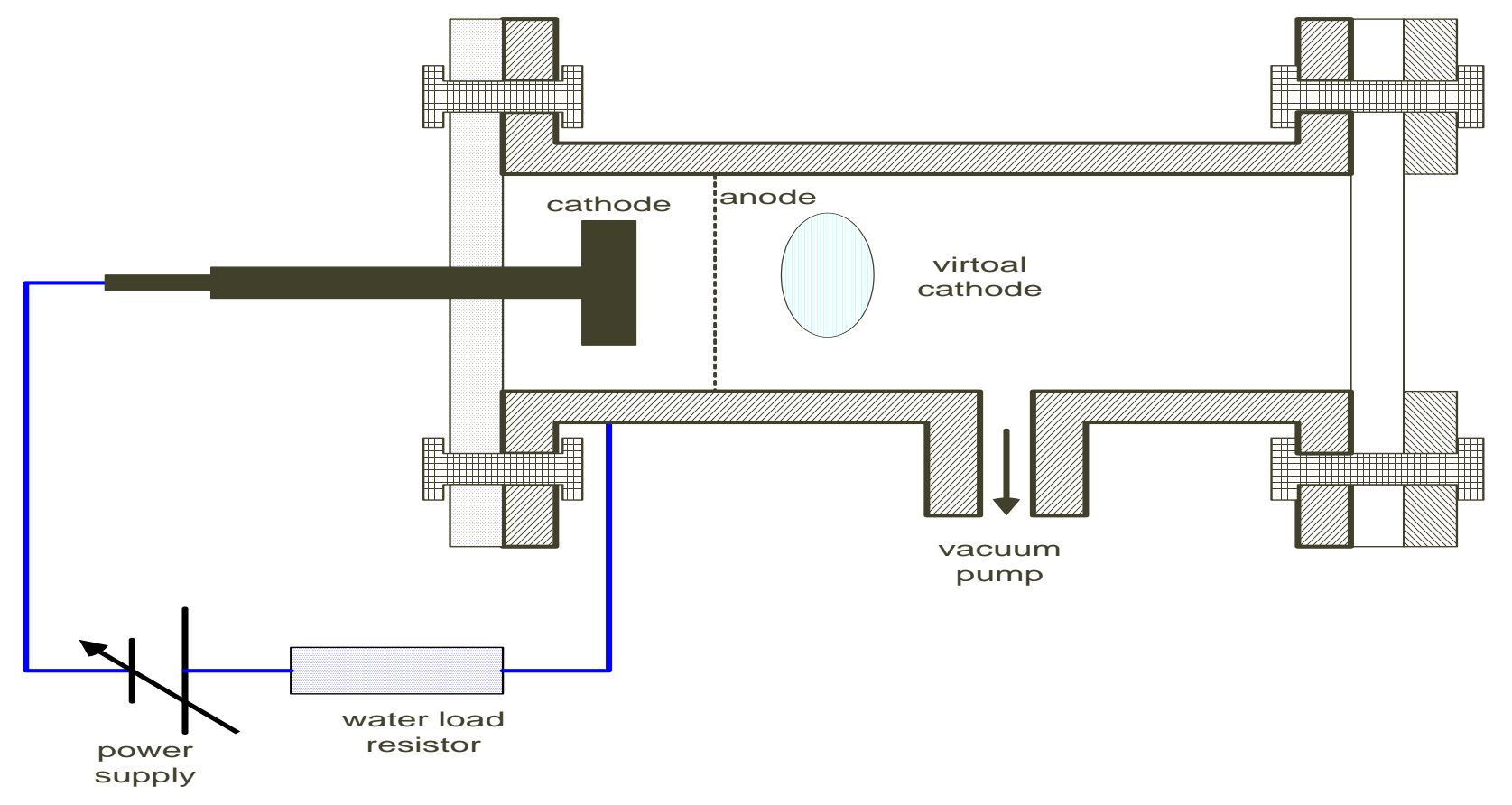

Fig. 1.Schematic diagram of vircator circuit with DC power supply

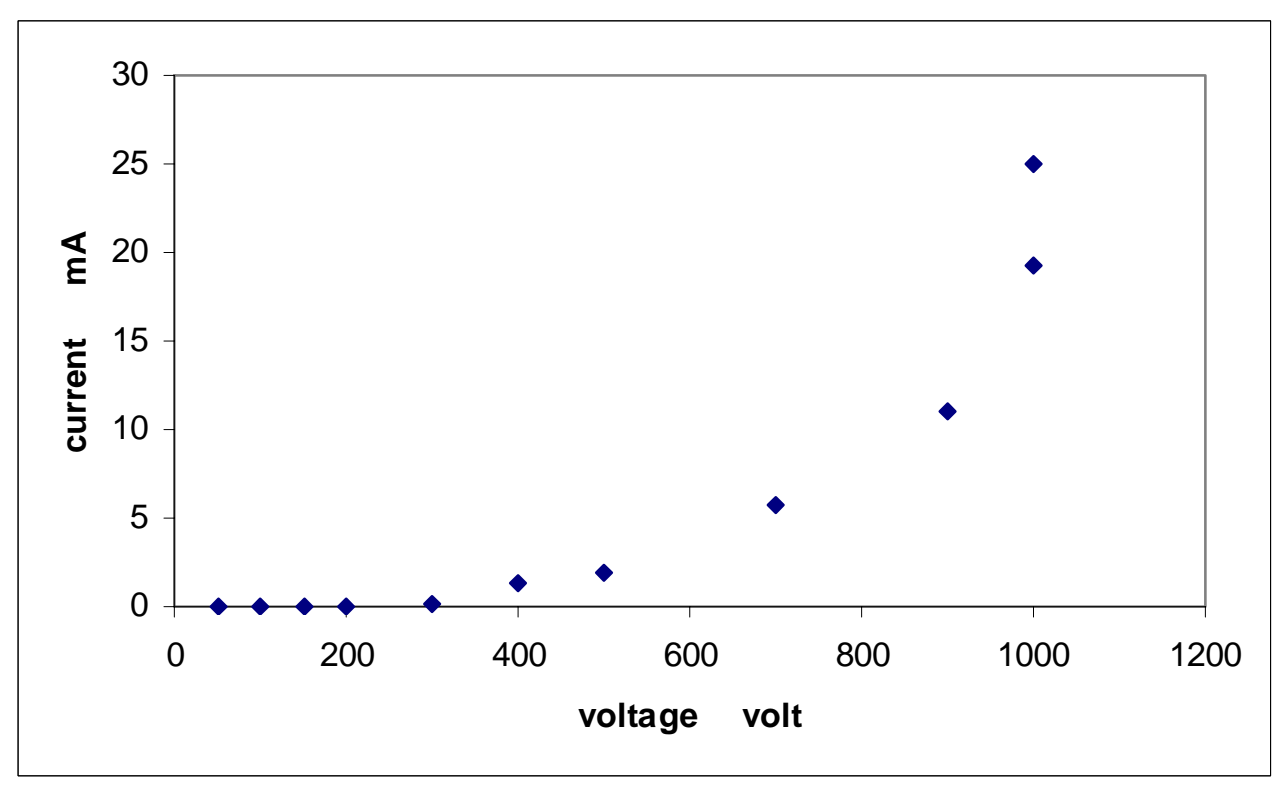

Fig. 2.I-V Characteristics of the discharge 


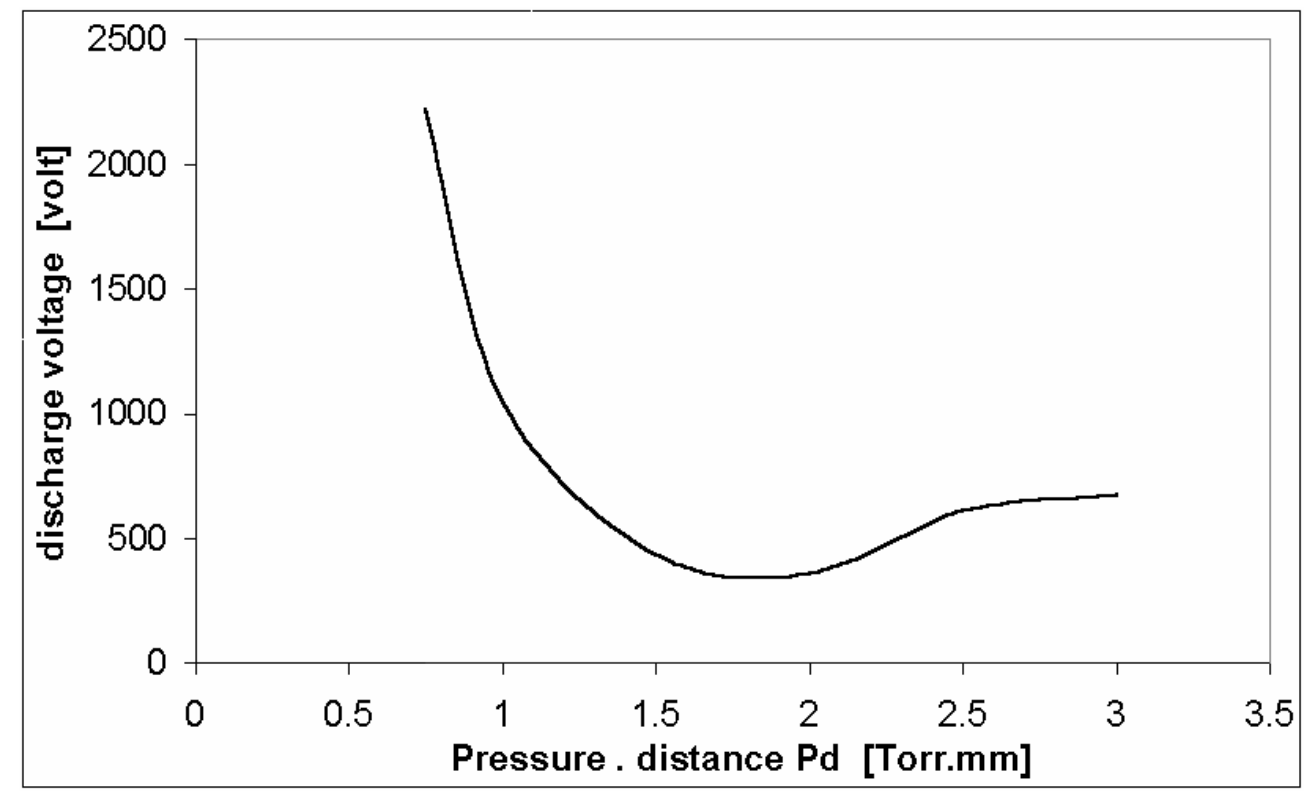

Fig. 3. Discharge voltage versus Pd

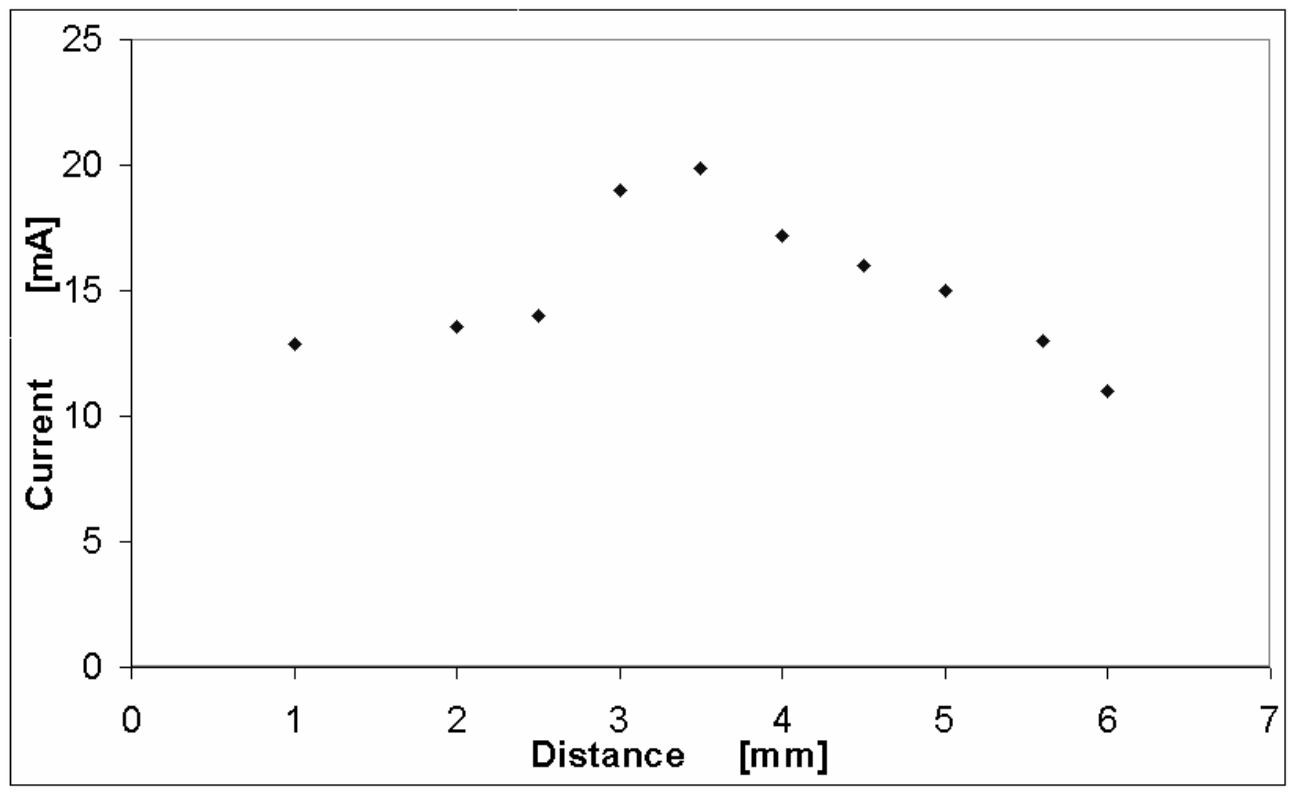

Fig. 4. Discharge electron beam current versus A-K spacing at pressure $5 \times 10^{-1}$ Torr, 


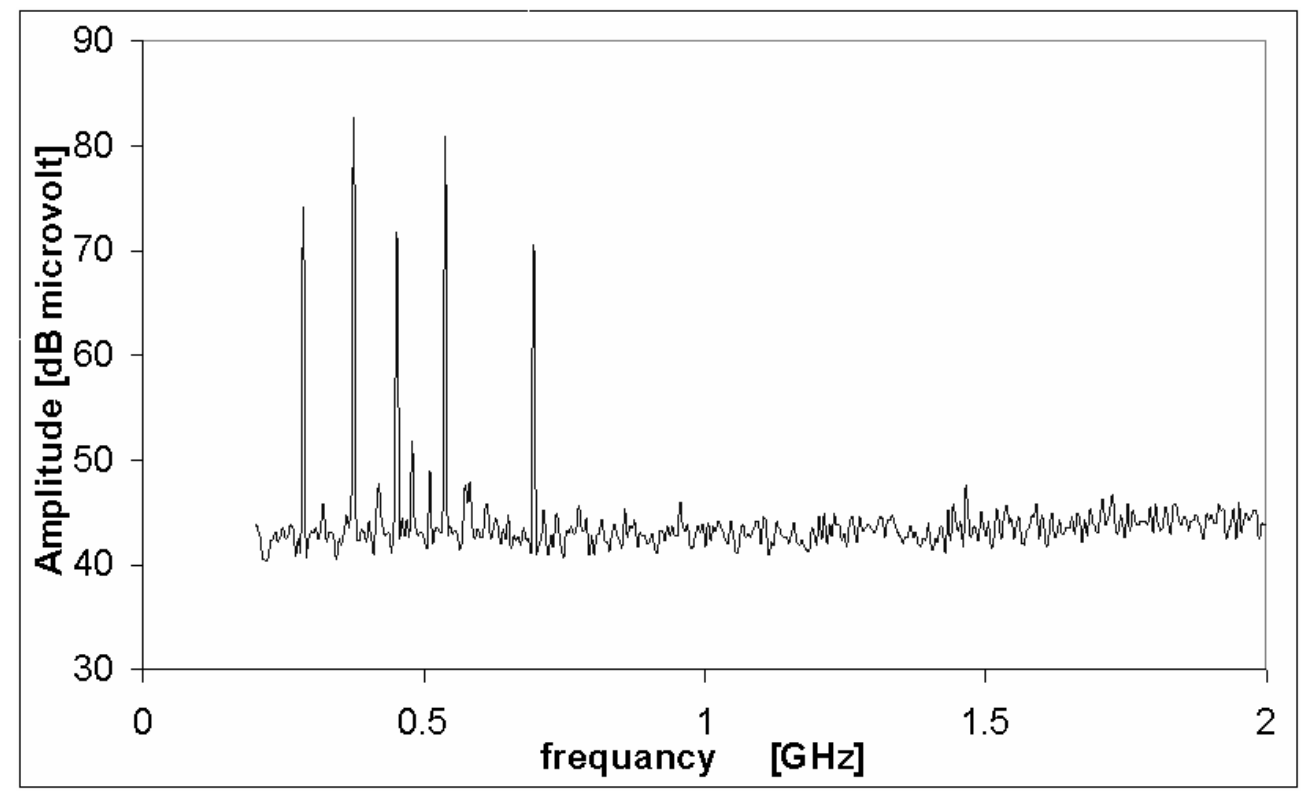

Fig. 5. The emitted microwave voltage amplitude versus frequency, by the frequency analyzer, from $D C$ vircator operated at working gas pressure $5 \times 10^{-1}$ Torr, discharge current $12.9 \mathrm{~mA}$, and A-K spacing of $3.5 \mathrm{~mm}$.

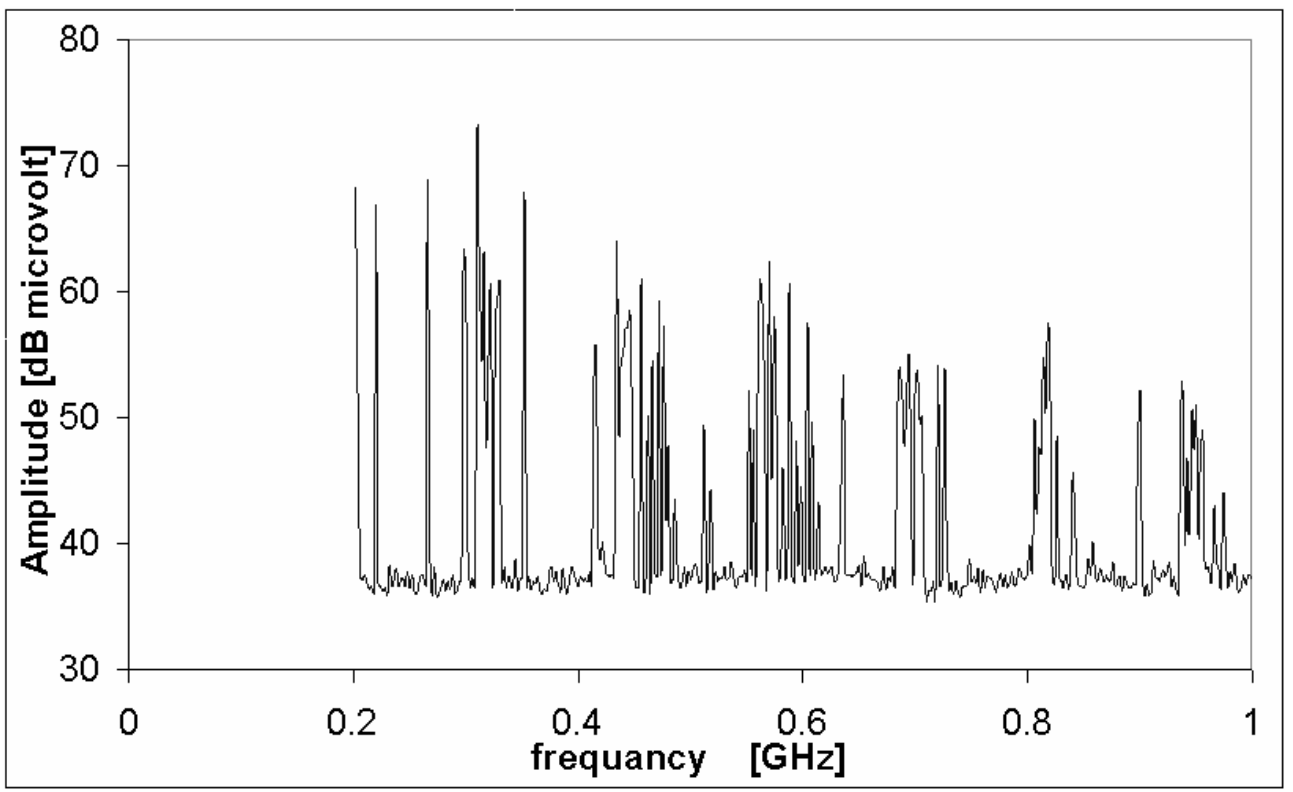

Fig. 6. The emitted microwave voltage amplitude versus frequency by the $D C$ vircator operated at working gas pressure $3 \times 10^{-1}$ Torr, discharge current $19.6 \mathrm{~mA}$, and A-K spacing of $3.5 \mathrm{~mm}$. 


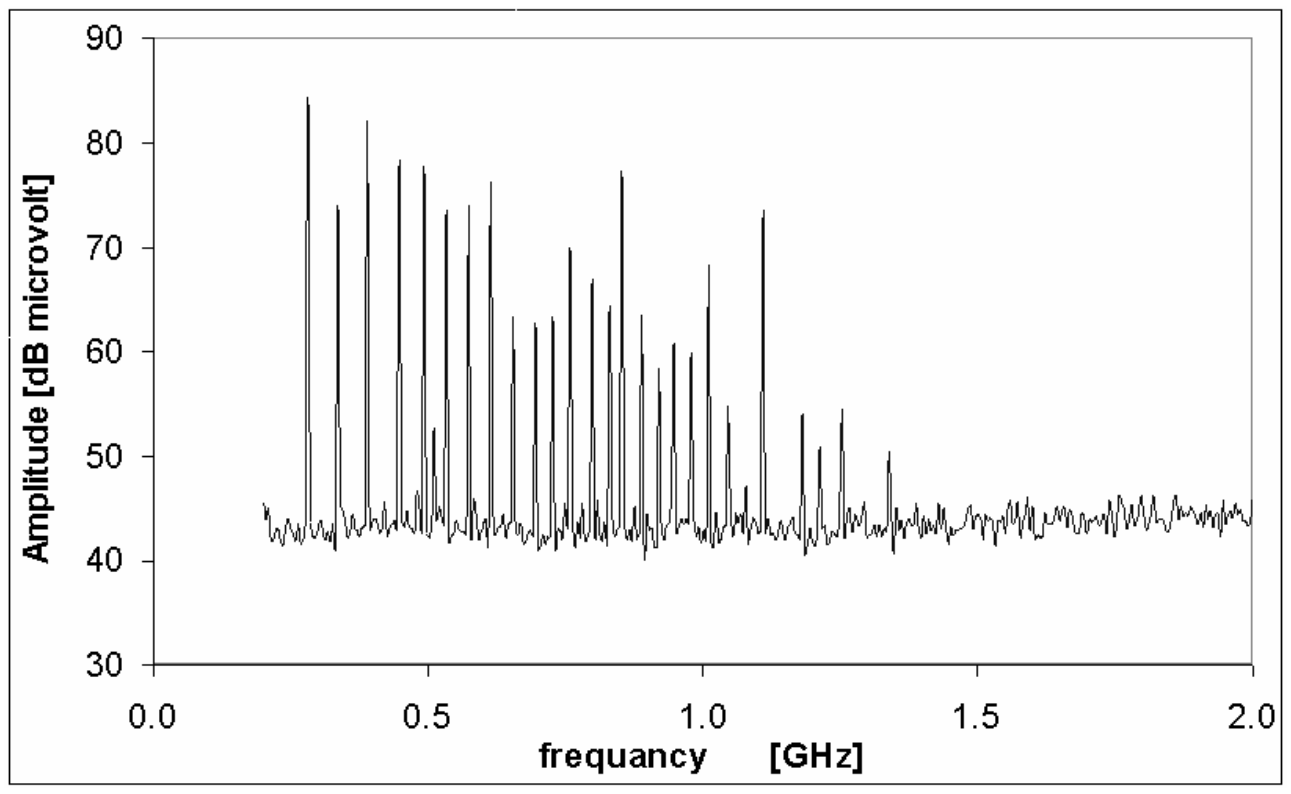

Fig. 7.The emitted microwave amplitude voltage versus frequency obtained at working gas pressures $7 \times 10^{-1}$ Torr, discharge current $15.5 \mathrm{~mA}$ for $3.5 \mathrm{~mm} \mathrm{A-K}$ spacing.

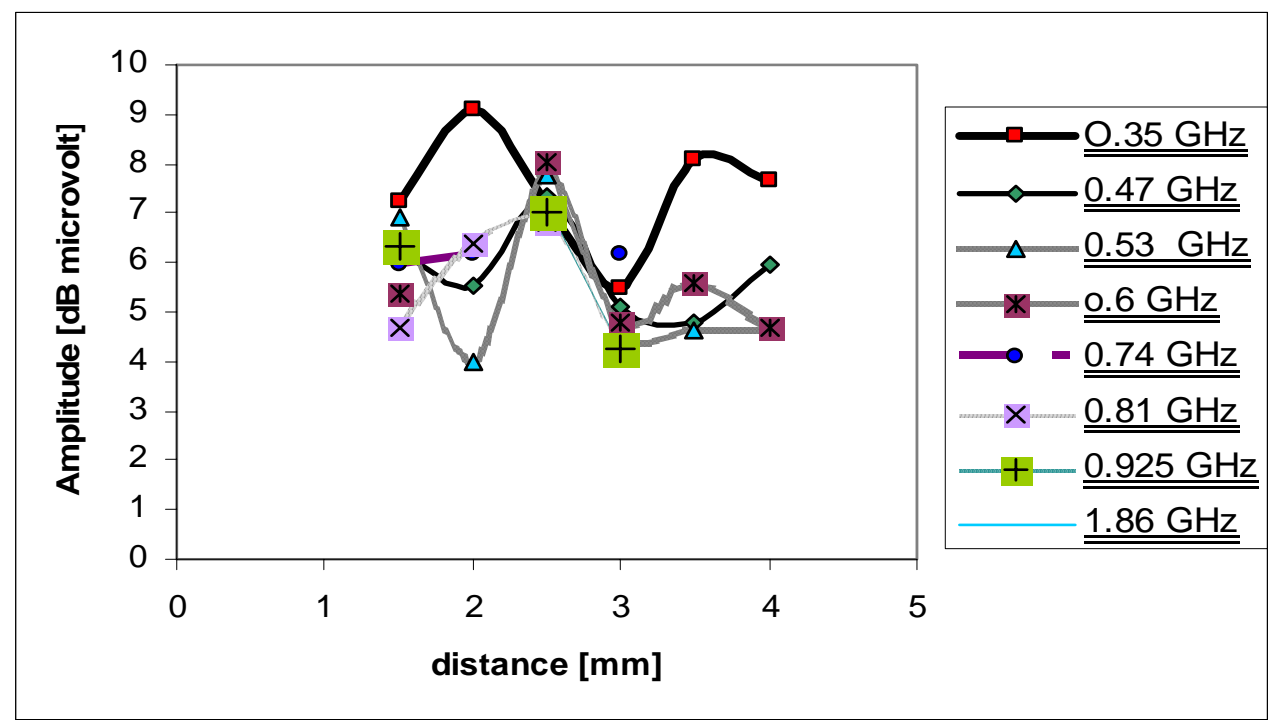

Fig. 8. Emitted wave amplitude voltage versus $A-K$ spacing for different frequencies at $P=5 \times 10^{-1}$ 


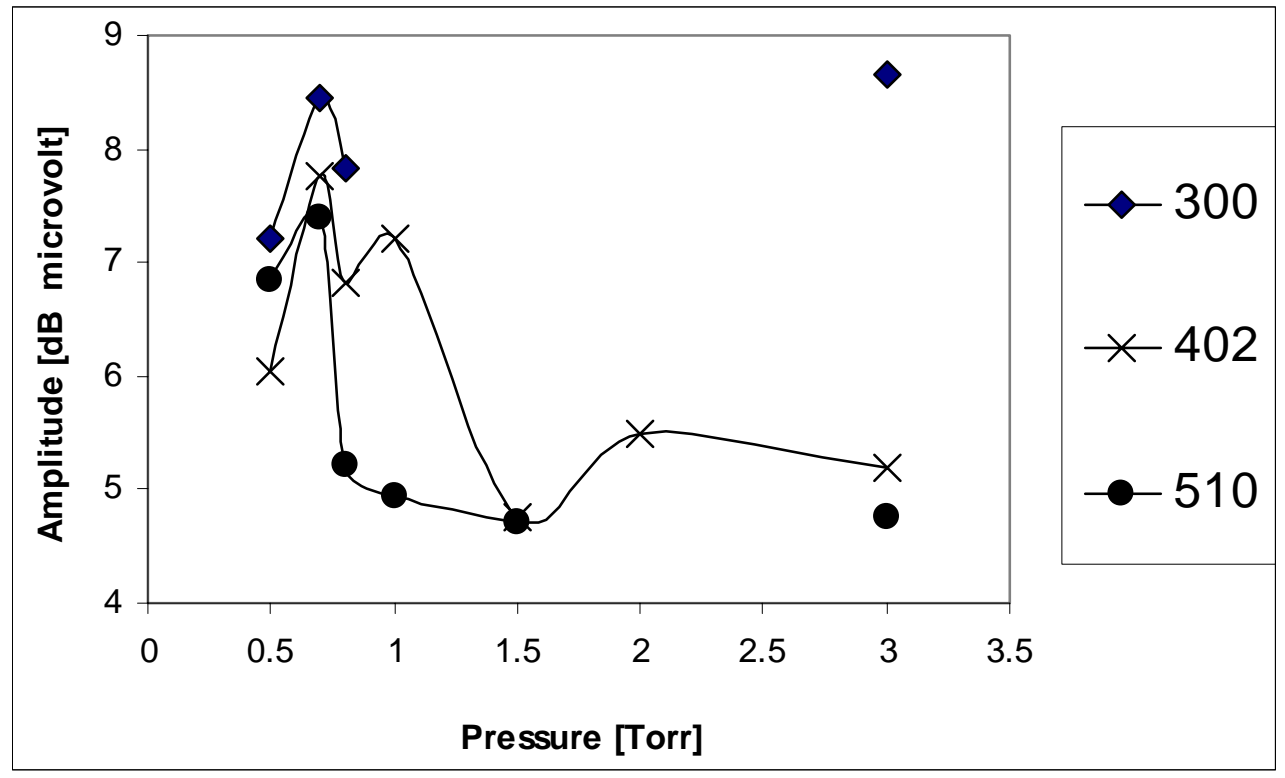

Fig. 9. Emitted wave amplitude voltage versus working gas pressure for different frequencies at $\mathrm{d}=3.5 \mathrm{~mm}$. 